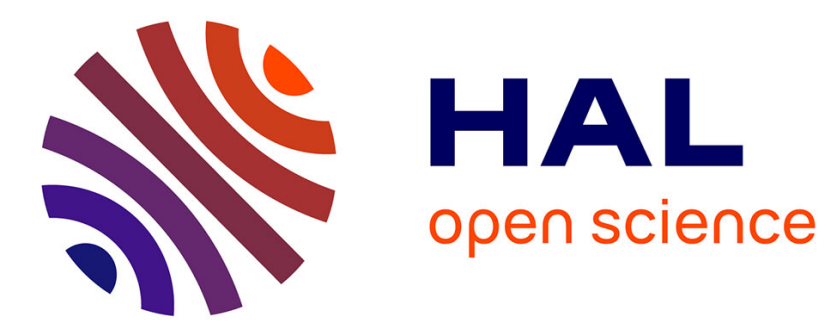

\title{
Generalization of the Q(ST) framework in hierarchically structured populations: Impacts of inbreeding and dominance
}

\author{
Philippe Cubry, Ivan Scotti, Sylvie Muratorio, Francois Lefèvre
}

\section{To cite this version:}

Philippe Cubry, Ivan Scotti, Sylvie Muratorio, Francois Lefèvre. Generalization of the Q(ST) framework in hierarchically structured populations: Impacts of inbreeding and dominance. Molecular Ecology Resources, 2017, 17 (6), pp.e76-e83. 10.1111/1755-0998.12693 . hal-01576329

\author{
HAL Id: hal-01576329 \\ https://hal.science/hal-01576329
}

Submitted on 25 May 2020

HAL is a multi-disciplinary open access archive for the deposit and dissemination of scientific research documents, whether they are published or not. The documents may come from teaching and research institutions in France or abroad, or from public or private research centers.
L'archive ouverte pluridisciplinaire HAL, est destinée au dépôt et à la diffusion de documents scientifiques de niveau recherche, publiés ou non, émanant des établissements d'enseignement et de recherche français ou étrangers, des laboratoires publics ou privés.

\section{(ㄷ)(1) $\$$}

Distributed under a Creative Commons Attribution - NonCommerciall 4.0 International 
1 Generalization of the $\boldsymbol{Q}_{s T}$ framework in hierarchically structured populations: impacts

2 of inbreeding and dominance.

3 Philippe Cubry ${ }^{1,2}$, Ivan Scotti ${ }^{1}$, Sylvie Oddou-Muratorio ${ }^{1}$, François Lefèvre ${ }^{1}$ *

4

$5 \quad{ }^{1}$ INRA, UR 629 Ecologie des Forêts Méditerranéennes, URFM, Domaine Saint Paul,

6 AgroParc, 84914 Avignon Cedex9, France

$7 \quad{ }^{2}$ IRD, UMR DIADE, 393 Avenue Agropolis, 34394 Montpellier, Cedex 5, France

8

9 Keywords: hierarchical Q-statistics, hierarchical F-statistics, quantitative genetics

10

11 * Corresponding author. Fax: +33.4.32722902 E-mail: $\underline{\text { francois.lefevre.2@inra.fr }}$

12

13 Running title: Generalized hierarchical Q-statistics

14

15 Abstract: 240 words

16 Total: 4570 words

$17 \quad 1$ table +2 figures 
18 Abstract

$19 Q_{\text {ST }}$ is a differentiation parameter based on the decomposition of the genetic variance of a trait.

20 In the case of additive inheritance and absence of selection, it is analogous to the genic

21 differentiation measured on individual loci, $F_{S T}$. Thus, $Q_{S T}-F_{S T}$ comparison is used to infer

22 selection: selective divergence when $Q_{S T}>F_{S T}$, or convergence when $Q_{S T}<F_{S T}$. The definition

23 of Q-statistics was extended to two-levels hierarchical population structures with Hardy-

24 Weinberg equilibrium. Here, we generalize the Q-statistics framework to any hierarchical

25 population structure. First, we developed the analytical definition of hierarchical Q-statistics

26 for populations not at Hardy-Weinberg equilibrium. We show that the Q-statistics values

27 obtained with the Hardy-Weinberg definition are lower than their corresponding F-statistics

28 when $F_{I S}>0$ (higher when $F_{I S}<0$ ). Then, we used an island model simulation approach to

29 investigate the impact of inbreeding and dominance on the $Q_{S T}-F_{S T}$ framework in a

30 hierarchical population structure. We show that, while differentiation at the lower hierarchical

31 level $\left(Q_{S R}\right)$ is a monotonic function of migration, differentiation at the upper level $\left(Q_{R T}\right)$ is not.

32 In the case of additive inheritance, we show that inbreeding inflates the variance of $Q_{R T}$, which

33 can increase the frequency of $Q_{R T}>F_{R T}$ cases. We also show that dominance drastically

34 reduces Q-statistics below F-statistics for any level of the hierarchy. Therefore, high values of

35 Q-statistics are good indicators of selection, but low values are not in the case of dominance. 


\section{Introduction}

39 Quantitative measures of adaptive genetic differentiation are needed for the study of local

40 adaptation processes. The Qst statistic based on a partition of the genetic variance between

41 and within populations is broadly used to quantify populations differentiation at quantitative

42 traits in simple population structures. Its comparison to neutral marker divergence, measured

43 via $F_{S T}$, provides a useful tool to understand the processes that lead to differentiation (see

44 Leinonen et al. 2008 for review). In the case of neutrality, linkage equilibrium, Hardy-

45 Weinberg equilibrium and additive inheritance, the mean value of differentiation at trait level,

$46 Q_{S T}$, equals the mean value of differentiation at gene level, $F_{S T}$, and their variances have the

47 same order of magnitude (Whitlock 2008). In other words, the distribution of single locus $F_{S T}$

48 values provides a reference to which $Q_{S T}$ values may be compared to infer the occurrence of

49 selection leading to divergence (high values of $Q_{S T}$ ) or convergence (low values of $Q_{S T}$ ).

50 Leinonen et al. (2013) highlighted the usefulness of the $Q_{S T}-F_{S T}$ comparison approach for

51 detecting adaptive evolution in various ecological contexts. In particular, this is expected to be

52 a powerful approach to detect recent adaptation because $Q_{S T}$ is partly determined by

53 covariances among QTL, which respond to selection more rapidly than individual allele

54 frequencies (Latta 1998).

55 The $Q_{S T}-F_{S T}$ comparison framework was initially defined assuming strictly additive

56 inheritance of the quantitative trait, Hardy-Weinberg equilibrium, absence of structured

57 linkage disequilibrium (LD), and absence of selection. Violations of these assumptions have a

58 variety of effects. Goudet \& Büchi (2006) and Goudet \& Martin (2007) showed that

59 dominance generally reduces the mean value of $Q_{S T}$ below the mean value of $F_{S T}$ in the island 
60 model, although the exact outcome may depend on demographic history and migration rates

61 among demes (see Whitlock 2008 for review). Whitlock (1999) and López-Fanjul et al.

62 (2003) showed that additive-by-additive epistasis tends to have the same effect in general.

63 Thus, with non-additive inheritance, the $Q_{S T}-F_{S T}$ approach is conservative in detecting

64 selection leading to adaptive divergence, but liberal in detecting selection leading to adaptive

65 convergence (Whitlock 2008). Relaxing the Hardy-Weinberg hypothesis, Bonnin et al. (1996)

66 extended the definition of $Q_{S T}$ for inbred populations, aiming to keep the property of equal

67 mean values of $Q_{S T}$ and $F_{S T}$ in that case. Regarding the assumption on LD, Whitlock (2008)

68 argued that, in the absence of selection, random LDs for different sets of loci compensate one

69 another and, therefore, have no effect on mean $Q_{S T}$ for neutral traits. Selection induces non-

70 independence among QTL and makes expected $Q_{S T}$ deviate from single-locus $F_{S T}$. Gavrilets \&

71 Hasting (1995) proposed an analytical framework to investigate the impact of QTL covariance

72 on $Q_{\text {ST }}$ at each level of the population structure. Kremer \& Le Corre (2012) quantified the

73 decoupling between $Q_{S T}$ and $F_{S T}$ under different evolutionary scenarios in a simple (non

74 hierarchical) population structure. Santure \& Wang (2009) showed that dominance and

75 inbreeding generally inflate the decoupling between $Q_{S T}$ and $F_{S T}$.

76 Besides one-level population structures, hierarchical structures attract increasing interest in

77 ecology (Richardson et al. 2014). Whitlock \& Gilbert (2012) stressed the interest of detecting

78 selection across differing levels in spatial hierarchical population structures with varying

79 amounts of gene flow. A hierarchical population structure may also result from temporal

80 patterns of gene flow, e.g. across cohorts within populations. It also occurs in plant or animal

81 breeding programs, e.g. sublining breeding schemes. More generally, understanding

82 evolutionary processes in hierarchical population models helps interpret situations where 
83 undetected within-population substructure may be suspected, e.g. in case of high within-

84 population environmental heterogeneity leading to microgeographic adaptation (Scotti et al.

85 2016).

86 Whitlock \& Gilbert (2012) introduced a hierarchical extension of Q-statistics measuring

87 genetic differentiation at different spatial scales in a hierarchical island model or, more

88 generally, for each level of any hierarchical population structure. Using the hierarchical

89 derivations of F-statistics from Slatkin \& Voelm (1991) and Yang (1998), it is then possible to

90 perform Q-statistics - F-statistics comparisons for each hierarchical level of the population

91 structure. However, these definitions of hierarchical Q-statistics do not account for local

92 departure from Hardy-Weinberg equilibrium, such as inbreeding, and the impact of

93 dominance in hierarchical population structure has not yet been investigated.

94 Here, we extend the framework of $Q_{S T}-F_{S T}$ comparison in hierarchical population structures

95 with no selection. We first summarize previous analytical developments that led to the

96 definition of hierarchical Q-statistics in Hardy-Weinberg populations and generalize these

97 derivations to account for inbreeding. Based on these developments, we analyze the effect of

98 inbreeding on $Q$-statistics and the consequences of ignoring it on $Q_{S T}-F_{S T}$ comparisons.

99 Next, we present the results of simulations in a hierarchical island model and analyze the

100 impacts of dominance on (i) the Q-statistics and (ii) the relation between Q-statistics and F-

101 statistics for each level of the hierarchy.

103 1) Definition of hierarchical Q-statistics for populations at Hardy-Weinberg equilibrium:

104 a synthesis 
106 In the case of a single gene, which extends to the case of additive polygenic inheritance with

107 no LD by summing over loci, Wright (1951) showed how overall departure from Hardy-

108 Weinberg equilibrium in a mixture of populations having different allele frequencies affects

109 genetic variance. In a diploid organism, the additive genetic variance in the total population

$110\left(\sigma_{I T}^{2}\right)$ is related to the one that would be achieved in case of Hardy-Weinberg equilibrium

$111\left(\sigma_{T(0)}^{2}\right)$ through the fixation index $F$ :

$112 \quad \sigma_{I T}^{2}=(1+F) \sigma_{T(0}^{2}$

113 Wright also demonstrated that the average within-population variance $\sigma_{I S}^{2}$ relates to $\sigma_{T(0)}^{2}$ :

$114 \quad \sigma_{I S}^{2}=(1-F) \sigma_{T(0)}^{2}(\mathrm{Eq} 2)$.

115 Therefore, due to the additivity of variance components, Wright deduced the variance among

116 populations $\left(\sigma_{S T}^{2}\right)$ from the difference between the total variance and the within-population

117 variance:

118

$$
\sigma_{S T}^{2}=(1+F) \sigma_{T(0)}^{2}-(1-F) \sigma_{T(0)}^{2}=2 F \sigma_{T(0)}^{2}(\mathrm{Eq} 3) .
$$

119 Considering here populations at Hardy-Weinberg equilibrium $\left(F_{I S}=0\right)$, the genic

120 differentiation between populations is $F=F_{I T}=F_{S T}$. Lande (1992) resolved these equations

121 for $F_{S T}$ : 
123 Spitze (1993) called $Q_{S T}$ this estimator of genic differentiation based on the variance

124 components. We will further call it $Q_{S T(0)}$ to indicate that it refers to the particular case where

$125 \quad F_{I S}=0$.

\section{6 . Extension to a hierarchical population structure}

127 The hierarchical extension of $F_{S T}$ has been described by several authors (Slatkin \& Voelm 128 1991; Yang 1998). In order to enable $Q_{S T}-F_{S T}$ comparisons at any level of population 129 structure, Whitlock \& Gilbert (2012) proposed a hierarchical extension of $Q_{S T(0)}$. To do so,

130 they introduced a regional level (R) of structure, above the population level. The

131 corresponding variance component, called $V_{R}$ by the authors, is noted here $\sigma_{R T}^{2}$ to follow 132 Wright's notation.

133 Following Wright's partitioning of variances in Eq 3, Whitlock \& Gilbert (2012) wrote that

$134 \quad \sigma_{R T}^{2}=2 F_{R T} \sigma_{T(0)}^{2}(\mathrm{Eq} 5)$.

135 Using Eq 2, they also gave the within-region component of variance as

$136 \quad \sigma_{W R}^{2}=\left(1-F_{R T}\right) \sigma_{T(0)}^{2}(\mathrm{Eq} 6)$.

137 For each region, between-population $\left(\sigma_{S R}^{2}\right.$, noted $V_{B}$ in Whitlock \& Gilbert 2012) and within-

138 population $\left(\sigma_{\text {IS }}^{2}\right.$ or $\left.V_{A}\right)$ components of genetic variance can then be written as: 


$$
\sigma_{S R}^{2}=2 F_{S R} \sigma_{W R}^{2}=2 F_{S R}\left(1-F_{R T}\right) \sigma_{T(0)}^{2} \quad(\mathrm{Eq} 7),
$$

$$
\sigma_{I S}^{2}=\left(1-F_{S R}\right) \sigma_{W R}^{2}=\left(1-F_{S R}\right)\left(1-F_{R T}\right) \sigma_{T(0)}^{2} \quad(\mathrm{Eq} 8) .
$$

$141 \quad$ Expressing $\sigma_{S R}^{2}$ in terms of $\sigma^{2}$ IS led to

$$
\sigma_{S R}^{2}=2 F_{S R} \frac{\sigma_{I S}^{2}}{\left(1-F_{S R}\right)} \text { (Eq 9), }
$$

143 Eq 9 provides a quantitative differentiation parameter for the lowest level of the hierarchy,

$144 Q_{S R(0)}$, based on the variance components:

$$
Q_{S R(0)}=F_{S R}=\frac{\sigma_{S R}^{2}}{\sigma_{S R}^{2}+2 \sigma_{I S}^{2}} \quad(\text { Eq 10), }
$$

146 which is the same as Eq 4 for the lowest hierarchical level in the population structure.

147 For the differentiation at the highest level of the hierarchy, Whitlock \& Gilbert (2012)

148 expressed $\sigma_{R T}^{2}$ in terms of $\sigma_{S R}^{2}$ :

$$
\sigma_{R T}^{2}=2 F_{R T} \sigma_{T(0)}^{2}=2 F_{R T} \frac{\sigma_{S R}^{2}}{\left(1-F_{R T} \mid 2 F_{S R}\right.} \quad(\text { Eq 11). }
$$

150 Resolving for $F_{R T}$ and using Eq 10 to replace $F_{S R}$ by its value, they defined $Q_{R T(0)}$ as follows:

$$
Q_{R T(0)}=F_{R T}=\frac{\sigma_{R T}^{2}}{\sigma_{R T}^{2}+\sigma_{S R}^{2}+2 \sigma_{I S}^{2}} \quad(\text { Eq 12). }
$$


152 Similarly, Q-statistics can be extended to any level of hierarchy (demonstration given in 153 supporting information S1). If we note the hierarchical levels 1 to $\mathrm{n}$, from lowest to highest

154 ( $\mathrm{n}=1$ in a simple population structure), we get:

$$
Q_{n(0)}=F_{n}=\frac{\sigma_{n}^{2}}{\left[\sum_{i=1}^{n} \sigma_{i}^{2}\right]+2 \sigma_{I S}^{2}}
$$

2) Generalized definitions of $Q$-statistics when $F_{I S} \neq 0$

\section{Simple population structure}

159 Wright (1951) generalized Eq 1-3 to relax the assumption of Hardy-Weinberg equilibrium

160 within sub-populations (positive or negative $F_{I S}$ ). In this case, the F-statistics are no longer the 161 same $\left(F_{I S} \neq 0\right.$ and $\left.F_{I T} \neq F_{S T}\right)$. Accordingly, Wright derived the components of genetic variance,

162 noted $\sigma_{I S(f)}^{2}, \sigma_{S T(f)}^{2}$ and $\sigma_{I T(f)}^{2}$, as follows. Considering that departure from Hardy-Weinberg

163 within populations does not affect the variance between populations, $\sigma_{S T(f)}^{2}$ remains the same

164 as $\sigma_{S T}^{2}: \sigma_{S T(f)}^{2}=2 F_{S T} \sigma_{T(0)}^{2}$. At the level of the whole population, we now have:

166 previous equations: $\sigma_{I S(f)}^{2}=\left(1+F_{I T}-2 F_{S T}\right) \sigma_{T(0)}^{2}$.

167 Replacing $F_{I T}$ by its expression in terms of $F_{S T}$ and $F_{I S}\left(F_{I T}=F_{S T}+F_{I S}-F_{S T} F_{I S}\right)$ led to

$$
\sigma_{I S(f)}^{2}=\left(1+F_{I S}\right)\left(1-F_{S T}\right) \sigma_{T(0)}^{2}(\mathrm{Eq} 14) .
$$


169 Therefore, using Eq 3 and Eq 14, Bonnin et al. (1996) derived a generalized relation for $Q_{S T(f)}$

170 in case of inbred populations (the $f$ subscript indicates that any possible value of $F_{I S}$ is now

171 taken into account):

172

$$
Q_{S T(f)}=F_{S T}=\frac{\left(1+F_{I S}\right) \sigma_{S T(f)}^{2}}{\left(1+F_{I S}\right) \sigma_{S T(f)}^{2}+2 \sigma_{I S(f)}^{2}} \quad \text { (Eq 15). }
$$

173 For $F_{I S}=0$, this general equation simplifies to the most commonly used $Q_{\text {ST(0). }}$.

\section{4 . Extension to a hierarchical population structure}

175 Here, we follow Bonnin et al. (1996) and generalize the hierarchical $Q_{R T(0)}$ and $Q S T(0)$ to the 176 case of populations not at Hardy-Weinberg equilibrium. Following Wright's demonstration, 177 the variance between regions is not affected by population structure, thus Eq 5 still holds and $178 \sigma_{R T(f)}^{2}=\sigma_{R T(0)}^{2}=2 F_{R T} \sigma_{T(0)}^{2}$. We now have to introduce two useful parameters: $\sigma_{W R(0) \text {, which is }}^{2}$ 179 the total genetic variance within regions if they were at Hardy-Weinberg equilibrium (i.e. 180 analog to $\sigma_{T(0)}^{2}$ but for the within-region additive genetic variance), and $F_{I R}$, which is the $F$ 181 statistic corresponding to individual relative to region level, i.e. the global fixation index 182 within region. $F_{I R}$ relates to $F_{S R}$ and $F_{I S}$ through the relation: $F_{I R}=F_{S R}+F_{I S}-F_{S R} F_{I S}$.

$183 \sigma_{W R(0)}^{2}$ is the one defined in Eq 6, whereas actual $\sigma_{W R(f)}^{2}$ relates to $\sigma^{2}{ }_{W R(0)}$ following Wright's 184 equations:

$$
\sigma_{W R(f)}^{2}=\left(1+F_{I R}\right) \sigma_{W R(0)}^{2}=\left(1+F_{I R}\right)\left(1-F_{R T}\right) \sigma_{T(0)}^{2} \quad(\text { Eq 16). }
$$

186 Following Eq 14 and Eq 6, $\sigma_{\text {IS(f) }}^{2}$ can be written in terms of $F_{I S}$ and $F_{S R}$ : 
$188 \sigma_{S R(f)}^{2}$ can be written as: $\sigma_{S R(f)}^{2}=2 F_{S R} \sigma_{W R(0)}^{2}=2 F_{S R}\left(1-F_{R T}\right) \sigma_{T(0)}^{2} \quad$ (Eq 18).

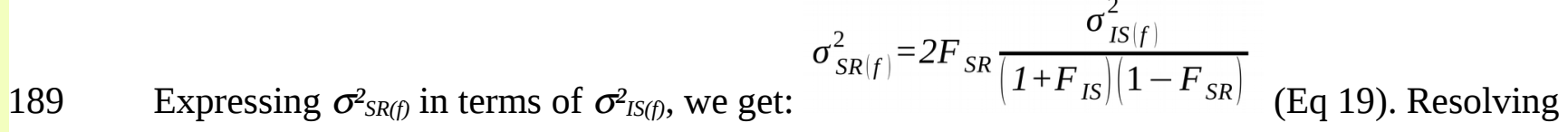

190 this equation for $F_{S R}$ gives the generalized definition of $Q S R(f)$ when populations are not

191 panmictic:

$$
Q_{S R(f)}=F_{S R}=\frac{\left(1+F_{I S}\right) \sigma_{S R(f)}^{2}}{\left(1+F_{I S}\right) \sigma_{S R(f)}^{2}+2 \sigma_{I S(f)}^{2}} \quad \text { (Eq 20), which is equivalent to Eq } 15 .
$$

$193 Q_{R T(f)}$ is calculated accordingly, replacing $F_{S R}$ by its value in Eq 11 for $\sigma_{R T}^{2}$ :

$$
\sigma_{R T(f)}^{2}=2 F_{R T} \frac{\sigma_{S R(f)}^{2}}{2\left(1-F_{R T}\right) \frac{\left(1+F_{I S}\right) \sigma_{S R(f)}^{2}}{\left(1+F_{I S}\right) \sigma_{S R(f)}^{2}+2 \sigma_{I S(f)}^{2}}}(\text { Eq 21). }
$$

195 Resolving this for $F_{R T}$ gives the generalized definition of $Q_{R T(f) \text { : }}$

$$
Q_{R T(f)}=F_{R T}=\frac{\left(1+F_{I S}\right) \sigma_{R T(f)}^{2}}{\left(1+F_{I S}\right) \sigma_{R T(f)}^{2}+\left(1+F_{I S}\right) \sigma_{S R(f)}^{2}+2 \sigma_{I S \mid f)}^{2}} \quad(\text { Eq 22). }
$$

197 This formula extends to any level of hierarchical structure as follows (demonstration given in 198 supporting information S1): 


$$
Q_{n(f)}=F_{n}=\frac{\left(1+F_{I S}\right) \sigma_{n}^{2}}{\left[\sum_{i=1}^{n}\left(1+F_{I S}\right) \sigma_{i}^{2}\right]+2 \sigma_{I S}^{2}}
$$

200 Table 1 summarizes the generalized formulae of the Q-statistics that are unbiased analogs of

$201 \quad F_{S T}$ when $F_{I S} \neq 0$, for any number of levels in population structure.

202 In empirical studies, the specific definitions of Q-statistics that assume Hardy-Weinberg

203 equilibrium $\left(Q_{S T(0)}\right)$ are more often used than the generalized definitions $\left(Q_{S T(f)}\right)$. In cases where

$204 F_{I S} \neq 0$ for whatever reason, $Q_{S T(0)}$ is a biased analog of $F_{S T}$, which may affect the $Q_{S T}-F_{S T}$

205 comparison approach. To quantify this bias, replacing $\sigma_{I S}^{2}$ and $\sigma_{S T}^{2}$ by their values (Eq 3 and

Eq 14, respectively) in Eq 15 leads to:

$$
Q_{S T(0)}=\frac{F_{S T}}{1+F_{I S}\left(1-F_{S T}\right)} \text {, which is also valid for } Q_{S R(0)} .
$$

207 Similarly, it results from Eq 22 that: $Q_{R T(0)}=\frac{F_{R T}}{1+F_{I S}\left(1-F_{S R}\right)\left(1-F_{R T}\right)}$. Since $1-F_{S T}, 1-F_{S R}$

208 and $1-F_{R T}$ are all positive, these relations show that neglecting departure from Hardy-

209 Weinberg equilibrium in the computation of Q-statistics provide lower values than the related

$210 \quad F$-statistics in case of inbreeding $\left(F_{I S}>0\right)$ or higher values in case of excess of heterozygotes

$211\left(F_{I S}<0\right)$. The difference is larger for intermediate values of differentiation. In a hierarchical

212 population structure, strong differentiation at lower levels (e.g high $F_{S R}$ value) reduces the bias

213 at upper levels $\left(Q_{R T(0)}-F_{R T}\right)$. 


\section{3) Impact of dominance on the $Q_{S T}-F_{S T}$ comparison in hierarchical populations}

216 We used the quantiNEMO simulation platform (Neuenschwander et al. 2008) to simulate a

217 hierarchical island model made of five regions and four populations within each region, i.e. 20

218 populations in total, and 100 individuals per population. We used three hierarchical structures

219 where the ratio of within- to between-region migration was set to 6, 15 and 30, respectively.

220 In each hierarchical structure, our sets of parameter values were mostly similar to Goudet \&

221 Büchi (2006). We used four global migration rates: $m=0.002,0.01,0.05$ and 0.2. Selfing rate

222 was 0 or 0.8 , leading to $F_{I S}$ values around 0.65 at the end of the simulations. The genetic

223 architecture of the trait consisted in 100 QTL, 10 alleles per QTL with a k-allele mutation

224 model parameter set to 0.001 , and the variance of allelic effects was set to unity. The

225 genotypic value of an individual was computed as: $G=\sum_{l o c i} a_{i}+a_{i^{\prime}}+k_{i i^{\prime}}\left|a_{i}-a_{i^{\prime}}\right|$ where $a_{i}$ and

$226 a_{i}$, are the allelic effects and $k_{i i}$ is the dominance parameter. Inheritance was either purely

227 additive $\left(k_{i i},=0\right.$ for all allelic pairs) or with dominance ( $\mathrm{k}_{i i}$, drawn from a normal distribution of

228 mean 0 and variance 1 , thus allowing over-dominance and under-dominance). Default

229 parameters were used for the initial genotype frequencies, i.e. initial populations were

230 maximally polymorphic in respect to allele frequencies. The hierarchical population structure

231 was allowed to evolve 1000 generations. Each set of varying parameters (ratio of within- to

232 between-region migration, global migration rate, selfing rate and dominance) was replicated

23310 times, i.e. 480 simulations were run in total. Setting files are provided as supporting

234 information S2.

235 At the end of each simulation, inbreeding $\left(F_{I S}\right)$ and within-population additive variance $\left(\sigma_{I S(f)}^{2}\right)$ 
236 values were directly taken from quantiNEMO outputs. We used the matrix of genotypes of the 237 last generation to estimate the hierarchical F-statistics $\left(F_{R T}, F_{S R}\right)$ using hierfstat package in $\mathrm{R}$

238 version 3.3.1. We used the matrix of genotypic values to estimate the hierarchical components

239 of genetic variance $\left(\sigma_{R T(f)}^{2}, \sigma_{S R(f)}^{2}\right)$ from a random linear model and maximum likelihood 240 estimator using lme4 package in $\mathrm{R}$.

241 These simulations illustrate an interesting feature of differentiation in hierarchical population

242 structures. We present here the results obtained with a ratio of within- to between-region

243 migration set to 15 (more details are given in supporting information S3; similar analyses with

244 the other values of this ratio, 6 and 30, are provided in supporting information S4). By

245 definition, the differentiation at regional level, $Q_{R T}$, not only depends on the variance among

246 regions $\left(\sigma_{R T(f)}^{2}\right)$ but also on the variance between populations within regions $\left(\sigma_{S R(f)}^{2}\right.$, see Eq. 22

247 above). Migration jointly influences both levels of the hierarchy and its effect on $\sigma_{R T(f)}^{2}$ may

248 be counterbalanced by its effect on $\sigma_{S R(f)}^{2}$. Therefore, unlike $Q_{S R}$ (or $Q_{S T}$ in a simple population

249 structure), $Q_{R T}$ is not a monotonic function of migration. This clearly appears in Figure 1 in

250 the purely additive case : when migration is very low (here when $m=0.002$ ), global and

251 within-region differentiation ( $Q_{S T \text {.global }}$ and $Q_{S R}$, respectively) are maximized but differentiation

252 at regional level $\left(Q_{R T}\right)$ is reduced. In our simulations, differentiation at regional level was

253 maximum when migration rate was $\mathrm{m}=0.01$.

254 In the case of pure additivity, inbreeding marginally increases the mean values of hierarchical $255 \quad$ Q-statistics and increases more significantly the variance of $Q_{R T}$ (Figure 1). Dominance

256 drasticaly reduces Q-statistics, globally and for each hierarchical level (Figure 1). 
257 In simulations under pure additivity, the relation between Q-statistics and F-statistics

258 followed the expectation that mean $Q_{S R}$ equals mean $F_{S R}$ as in a simple population structure

259 (Figure 2). The relation was slightly different at regional level: in the absence of inbreeding,

260 mean $Q_{R T}$ was slightly lower that mean $F_{R T}$. This discrepancy reflects the presence of

261 covariance terms between alleles that would deserve further investigation. With inbreeding,

262 the variance of $Q_{R T}$ increased, thus increasing the number of occurrences where $Q_{R T}>F_{R T}$

263 (Figure 2). With dominance, Q-statistics were always lower than F-statistics at the global

264 level, which is consistent with the results obtained in a simple population structure by Goudet

265 and Büchi (2006), and this remained true for each hierarchical level irrespective of the

266 presence or absence of inbreeding (Figure 2).

267 The same qualitative patterns as in Figure 1 and Figure 2 were obtained with the other ratios

268 of within- to between-region migration (see supporting information S4). The only noticeable

269 quantitative difference was the non-linear effect of the migration rate on $Q_{R T}$, which co-varied

270 with the ratio of within- to between-region migration, less pronounced with the lowest ratio.

\section{$271 \quad$ Discussion}

272 In this note, we proposed generalized definitions of Q-statistics for hierarchical population

273 structures that keep the property of analogy to hierarchical F-statistics even when the lowest

274 level of the population structure is not at Hardy-Weinberg equilibrium, in case of neutrality.

275 This property is essential to infer selection from the $Q_{S T}-F_{S T}$ comparison, and these

276 generalized definitions should be preferred to the simpler ones that assume Hardy-Weinberg

277 equilibrium. In random mating populations, one generation of panmixia is theoretically

278 enough to restore equilibrium after disturbance and, therefore, we expect little departure from 
279 Hardy-Weinberg equilibrium in practice. However, inbred mating systems or unrevealed

280 population substructure may generate positive $F_{I S}$, while partial asexuality may generate

281 negative $F_{I S}$ (Stoeckel \& Masson 2014). In this study, we quantified the discrepancy between

$282 \quad Q$-statistics and F-statistics induced by the use of Q-statistics definitions that do not account

283 for an existing sub-structure in a population system, i.e. non-null $F_{I S}$ values. This leads to

284 under-estimate $Q$-statistics when $F_{\text {IS }}$ is positive, and vice-versa. Therefore, when positive $F_{\text {IS }}$

285 is neglected in the computation of $Q$-statistics, the inference of adaptive divergence when $Q$ -

286 statistics are significantly higher than F-statistics is conservative but, reversely, inference of

287 adaptive convergence cannot be concluded when Q-statistics are lower than F-statistics. The

288 reverse effect occurs with negative $F_{I S}$ values. Interestingly, in a hierarchical population

289 structure, high differentiation at the lowest hierarchical level reduces the bias due to

290 neglecting $F_{I S}$ on the upper level Q-statistics.

291 The analytical framework leading to the definition of generalized Q-statistics relies on the

292 assumptions of additivity and independence among neutral loci. Here, in a hierarchical island

293 model, we show that dominance drastically reduces hierarchical $Q$-statistics $\left(Q_{R T}\right.$, $\left.Q_{S R}\right)$ below

294 their related F-statistics $\left(F_{R T}, F_{S R}\right)$, as initially shown by Goudet \& Büchi (2006) and Goudet

295 \& Martin (2007) in a simple island model. Therefore, dominance makes the $Q_{S T}-F_{S T}$

296 comparison a conservative approach when inferring selective divergence from high $Q_{S T}$ values

297 but no conclusion can be drawn from low $Q_{S T}$ values. For empirical studies, it should be

298 noticed that, even in the absence of selection, historical and demographic processes can

299 generate random statistical dependencies among loci that are captured in the Q-statistics but

300 not in the F-statistics. These covariances contribute to the high variance of Q-statistics. In our

301 simulated hierarchical island model, discrepancies between Q-statistics and F-statistics were 
302 more likely at the upper level of the hierarchy $\left(Q_{R T}-F_{R T}\right)$, and the variance of $Q_{R T}$ was

303 particularly sensitive to inbreeding in the case of pure additivity. Therefore, the power to

304 detect selection from the $Q_{S T}-F_{S T}$ approach is lower between regions than within regions.

305 When there is dominance, the mean and the variance of Q-statistics are both reduced. To

306 account for these neutral sources of covariance among loci, Q-statistics should rather be

307 compared to measures of neutral differentiation patterns that account for these disequilibria,

308 such as the $C F_{\text {ST }}$ proposed by Kremer et al. (1997). Statistical developments (Martin et al,

3092008 ) and the recent availability of genome-wide resources have provided some clues for the

310 analysis of polygenic adaptation (Berg \& Coop 2014; Yeaman 2015; Stephan 2016).

311 In a hierarchical population structure, we showed a specific effect of interaction between

312 migration and genetic drift on the differentiation among "regions" (or any other kind of up-

313 level of the hierarchy, not necessarily due to spatial structure) that has interesting

314 consequences for the conservation of the total genetic diversity. The migration rate has a non-

315 monotonic effect on the differentiation among "regions". Very low migration rates induce

316 high differentiation among "populations” within-“regions”, which is also related to high gene

317 diversity at "regional” level (data not shown). In this case, “populations” become quasi-

318 independent samples submitted to genetic drift and each "region”, having several of these

319 independent “populations”, captures a good level of gene diversity, thus reducing the

320 divergence among "regions”. In other words, in a hierarchical population structure, the impact

321 of genetic drift on gene diversity at "regional” level can be reduced not only by high

322 migration rates but also by very low migration rates, and this effect is increased when the ratio

323 of within- to between-“region” migration is high. 


\section{Acknowledgement}

This work was supported by Agence Nationale de la Recherche (ANR) under the FLAG project ANR-12-ADAP-0007-01. We are grateful to Antoine Kremer, Thomas Boivin, Catherine Bastien, Leopoldo Sanchez, Vincent Segura and anonymous reviewers for their helpful comments.

\section{$329 \quad$ References}

Berg JJ, Coop G (2014) A population genetic signal of polygenic adaptation. PLoS Genet, 10, e1004412.

Bonnin I, Prosperi JM, Olivieri I (1996) Genetic markers and quantitative genetic variation in Medicago truncatula (Leguminosae): a comparative analysis of population structure. Genetics, 143, 1795-1805.

Gavrilets S, Hasting A (1995) Dynamics of polygenic variability under stabilizing selection, recombination, and drift. Genetical Research, 65, 63-74.

Goudet J, Büchi L (2006) The effects of dominance, regular inbreeding and sampling design on $Q_{S T}$, an estimator of population differentiation for quantitative traits. Genetics, 172,1337-1347.

Goudet J, Martin G (2007) Under neutrality, $Q_{S T} \leq F_{S T}$ when there is dominance in an island model. Genetics, 176,1371-1374..

Kremer A, Zanetto A, Ducousso A (1997) Multilocus and multitrait measures of differentiation for gene markers and phenotypic traits. Genetics, 145, 1229-1241.

Kremer A, Le Corre V (2012) Decoupling of differentiation between traits and their underlying genes in response to divergent selection. Heredity, 108, 375-385.

Lande R (1992) Neutral theory of quantitative genetic variance in an island model with local extinction and colonization. Evolution, 46, 381-389.

Latta RG (1998) Differentiation of allelic frequencies at quantitative trait loci affecting locally adaptive traits. The American Naturalist, 151, 283-292.

Leinonen T, O’Hara RB, Cano JM, Merilä J (2008) Comparative studies of quantitative trait and neutral marker divergence: a meta-analysis. Journal of Evolutionary Biology, 21, $1-17$.

Leinonen T, McCairns RJS, O’Hara RB, Merilä J (2013) $Q_{S T}-F_{S T}$ comparisons: evolutionary and ecological insights from genomic heterogeneity. Nature Review Genetics, 14, 179-190. 
López-Fanjul C, Fernández A, Toro MA (2003) The effect of neutral nonadditive gene action on the quantitative index of population divergence. Genetics, 164, 1627-1633.

Martin G, Chapuis E, Goudet J (2008) Multivariate Qst - Fst comparisons: a neutrality test for the evolution of the G matrix in structured populations. Genetics, 180, 2135-2149.

Neuenschwander S, Hospital F, Guillaume F, Goudet J (2008) quantiNEMO: an individualbased program to simulate quantitative traits with explicit genetic architecture in a dynamic metapopulation. Bioinformatics, 24, 1552-1553.

Richardson JL, Urban MC, Bolnick DI, Skelly DK (2014) Microgeographic adaptation and the spatial scale of evolution. Trends in Ecology and Evolution, 29, 165-176.

Santure AW, Wang J (2009). The joint effects of selection and dominance on the $Q_{S T}-F_{S T}$ contrast. Genetics, 181, 259-276.

Scotti I, Gonzalez-Martinez SC, Budde KB, Lalagüe H (2016). Fifty years of genetic studies: what to make of the large amounts of variation found within populations? Annals of Forest Science, 73, 69-75.

Slatkin M, Voelm L (1991) $F_{S T}$ in a hierarchical island model. Genetics, 127, 627-629.

Spitze K (1993) Population structure in Daphnia obtusa: quantitative genetic and allozymic variation. Genetics, 135, 367-374.

Stephan W (2016) Signatures of positive selection: from selective sweeps at individual loci to subtle allele frequency changes in polygenic adaptation. Molecular Ecology, 25, 7988.

Stoeckel S, Masson JP (2014) The exact distributions of $F_{I S}$ under partial asexuality in small finite populations with mutation. PLoS ONE, 9, e85228.

Whitlock MC (1999) Neutral additive variance in a metapopulation. Genetical Research, 74, 215-221.

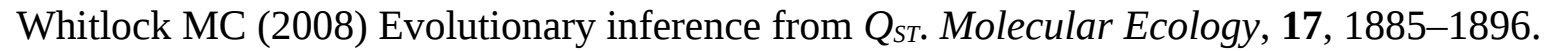

Whitlock MC, Gilbert KJ (2012) $Q_{S T}$ in a hierarchically structured population. Molecular Ecology Resources, 12, 481-483.

Wright S (1951) The genetical structure of populations. Annals of Eugenics, 15, 323-354.

Yang RC (1998) Estimating hierarchical F-Statistics. Evolution, 52, 950-956.

Yeaman S (2015) Local adaptation by alleles of small effect. The American Naturalist,. 186(Suppl), S74-S89. 
331 Detailed quantiNEMO parameter sets and simulation outputs are provided as supporting

332 information.

\section{$333 \quad$ Author contributions}

334 PC, IS, SOM and FL designed the research and wrote the paper. PC and FL made the 335 analytical developments and the simulations.

\section{$336 \quad$ Tables and Figures}

337 Table 1: Definition of generalized Q-statistics taking into account inbreeding in

338 hierarchically structured populations. See the main text for the definitions of the parameters.

339 Figure 1: Q-statistics in a hierarchical island model with five regions and four populations per 340 region. The ratio of within- to between-region migration was set to 15 and four migration 341 rates were used, with or without inbreeding, with or without dominance. Differentiation 342 parameters are computed globally $\left(Q_{\text {sT.global, }}\right.$, among the 20 populations), among regions $\left(Q_{R T}\right)$ 343 and among populations within regions $\left(Q_{S R}\right)$.

$344 \quad$ Figure 2: Relation between $Q$-statistics and F-statistics in the same hierarchical island model 345 as Figure 1 . Symbols reflect the migration rates $(0: m=0.002 ; \Delta: m=0.01 ;+: m=0.05 ; x$ : $346 \mathrm{~m}=0.2)$.

\section{$347 \quad$ Supporting information}

348 Supporting information S1: Demonstration of the generalized equations Eq 13 and Eq 23

349 Supporting information S2: Settings file used to elaborate 480 neutral evolutionary scenarios 350 in a two-levels hierarchical population structure with the quantiNEMO simulation platform.

351 Supporting information S3: Simulation outputs, diversity and differentiation parameters for 352 each simulation run with a ratio of within- to between-region set to 15.

$353 \quad$ Supporting S4: Additional results equivalent to Figure 1 and Figure 2 obtained with two other 354 ratios of within- to between-region migration, respectively set to 6 and 30 . 
Table 1

356

\begin{tabular}{|l|l|l|}
\hline Population structure & Definition of generalized hierarchical Q-statistics & Ref \\
\hline 1-level structure & $Q_{S T(f)}=\frac{\left(1+F_{I S}\right) \sigma_{S T(f)}^{2}}{\left(1+F_{I S}\right) \sigma_{S T(f)}^{2}+2 \sigma_{I S(f)}^{2}} \quad$ (Eq 15) & (1) \\
\hline$Q_{S R(f)}=\frac{\left(1+F_{I S}\right) \sigma_{S R(f)}^{2}}{\left(1+F_{I S}\right) \sigma_{S R(f)}^{2}+2 \sigma_{I S(f)}^{2}} \quad(\mathrm{Eq} 20)$ & $\left(1+F_{I S}\right) \sigma_{R T(f)}^{2}$ \\
\hline n-levels hierarchy & $Q_{R T(f)}=\frac{(2)}{\left(1+F_{I S}\right) \sigma_{R T(f)}^{2}+\left(1+F_{I S}\right) \sigma_{S R(f)}^{2}+2 \sigma_{I S(f)}^{2}} \quad$ (Eq 22) \\
$Q_{n(f)}=F_{n}=\frac{\left(1+F_{I S}\right) \sigma_{n}^{2}}{\left[\sum_{i=1}^{n}\left(1+F_{I S}\right) \sigma_{i}^{2}\right]+2 \sigma_{I S}^{2}}$
\end{tabular}

357 References: (1) Bonnin et al, 1996; (2) this paper (see text for other references that funded 358 these derivations) 